\title{
Heterogeneous Fleet Vehicle Routing Problem with Selec- tion of Inter-Depots
}

\author{
ČIČKOVÁ Zuzana, PEKÁR Juraj, BREZINA Ivan, REIFF Marian \\ Department of Operations Research and Econometrics, University of Economics, Bratislava, Slovakia \\ Email: pekar@euba.sk
}

Received 2012

\begin{abstract}
The paper presents heterogeneous fleet vehicle routing problem with selection of inter-depots. The goal of the proposed model is to schedule shipments of goods from the central depot to the inter-depots and finally to the endpoint customers, so that as inter-depots are considered the existing capacities of endpoints customers. In given type of transportation it can be expected to use two types of vehicles: large capacity vehicles ensuring the distribution of goods from the central warehouse and small capacity vehicles ensuring the delivery of goods from inter-depots for late shipment to other customers. The principle of the model is illustrated on three demonstrative instances
\end{abstract}

Keywords: multi-depot vehicle routing problem; replenishment; mathematical programming

\section{Introduction}

The problem that addresses the distribution of goods from a central warehouse to various delivery points using a fleet of vehicle with limited capacity is known as capacited vehicle routing problem (CVRP). The relevance of that problem follows from many published articles, and also from practical applications of distribution (e. g. [1], [2], [3], [8]). The CVRP belongs to the group of NP-hard problems, so the possibility of solution of above mentioned problem is studied by many authors. Nowadays research attention is focused on heuristics, for example, the evolutionary approach is proposed by in [2], [3], [4], [5], [6], [7]. The problem can be extended by adding inter-depots to the central storage facilities and also by considering the use of multiple types of vehicles ([6], [8], [9], [10], [11], [12]). Delivery is then realized in two levels, the first level is to carry the goods from a central distribution warehouse into inter-depots and the second level implements the transshipment of goods to the endpoint customers. Generally, each facility tends to be exactly defined to belong to the group of inter-depots or to the group of final customers. However, in nowadays real life situations, companies that are dealing with this type of distribution do not open new inter-depots, which opening is associated with the construction cost and subsequently with operation cost, but they utilize endpoint customers useful storage capacities also as an intermediate storage for late shipment to other customers. In this case, as inter-depots storage are used existing capacities of endpoints customers, so that existing supply points may or may not also serve as a inter-depots. In given type of problem it can be expected to use two types of vehicles: large capacity vehicles $(L)$ ensuring the distribution of goods from the central warehouse and small capacity vehicles $(S)$ ensuring the delivery of goods from inter-depots and also from central warehouse. In this paper we formulate the considered problem as a mathematical programming model later used for further analysis. Due to its computational complexity, the formulated problem is solvable by classical optimization techniques (as well as other modifications of CVRP) only for small instances. We chose the following structure of the paper: In the first part we present classical CVRP, because it forms a base of modeling for a wide variety of routing problems. The next section describes the construction of proposed heterogeneous fleet vehicle routing problem with selection of inter-depots. Afterwards, the obtained experimental results are given and discussed. In this section, we also analyze presented model under different vehicle cost.

\section{Capacited Vehicle Routing Problem}

Classical capacitated vehicle routing problem is the basic routing model and it can be stated as follows: Let $G=(V, E)$ be the full weighted graph, where $V$ represents set of $n$ nodes and $E$ represents $n . n$ set of edges. The node $v_{1}$ represents the location of central warehouse and the nodes $v_{i}=2,3, \ldots n$ represent location of endpoint customers.

Assumptions of CVRP:

- Unlimited number of vehicles with a certain capacity $g$, which are located in a certain depot with unlimited capacity. 


\section{Č. Zuzana $E T \quad A L$.}

- Set of delivery points (endpoints) with a certain demand $b_{i}, i=2,3, \ldots n$ (the orders have to be delivered in full).

- Minimal distances between all the pairs of endpoints customers and also between the endpoint customers and the depot are known (matrix D).

- The goal is to find optimal vehicle routes (so that the total distance traveled is minimized) in such a way that each customer is visited only once by exactly one vehicle, all routes start and end at the depot, and the total demands of all customers on one particular route must not exceed the capacity of the vehicle.

- In the model is implicitly assumed $b_{i} \leq g$ for each $i=2,3, \ldots n$.

\section{Parameters}

D - matrix of minimal distances between all the pairs of nodes

$c$ - transportation cost per unit

$\mathbf{b}-$ vector of demands

$g$ - capacity of the vehicle.

\section{Variables}

$x_{i j} \in\{0,1\}, i, j=1,2, . . n$ - binary variables, that represent the use of corresponding edge: If the edge $(i, j)$ is on the route of the some vehicle $x_{i j}=1$, otherwise $x_{i j}=0$,

$u_{i} \geq 0, i=1,2, \ldots n$ - non-negative variables, that represent the cumulative demand of the individual nodes on the route of any vehicle.

Starting value of variable

$u_{1}=0$

CVRP formulation can be interpreted as follows:

$$
f(\mathbf{X}, \mathbf{u})=c \sum_{i=1}^{n} \sum_{j=1}^{n} d_{i j} x_{i j} \rightarrow \min
$$

Objective function ensures minimization of total transportation costs of vehicles.

$\sum_{i=1}^{n} x_{i j}=1, j=2,3, \ldots n, i \neq j$

The equations represent that the vehicle comes to each node (except the centre) exactly once.

$\sum_{j=1}^{n} x_{i j}=1, i=2,3, \ldots n, i \neq j$

The equations represent that the vehicle leaves each node (except the centre) exactly once.

$\left(u_{i}+b_{j}-u_{j}\right) x_{i j}=0, \quad i=1,2, \ldots n, \quad j=2,3, \ldots n, \quad i \neq j$

$b_{i} \leq u_{i} \leq g, i=2,3, \ldots n$
The equations are anti-cyclic constraints which ensure also the accumulation of the load of the vehicle in such way the load must not exceed the vehicle capacity.

\section{Heterogeneous Fleet Vehicle Routing Problem with Selection of Inter-Depots - Problem Formulation}

The model was in general introduced by authors in [13]. Problem is useful for company dealing with one central warehouse (edge $v_{1}$ denotes localization of the central warehouse) and $n-1$ delivery points. The large capacity vehicles $L$ are available in the central warehouse. The small capacity vehicles $S$ are available at the endpoints and also at the central warehouse. Transportation costs per unit are known for both types of vehicles. It is assumed the known demand of all delivery points. All the orders have to be delivered in full. Each of the $n-1$ delivery point can be used as a inter-depot, so demand of other delivery point can be can be met from the inter-depot by vehicle $S$. The route of vehicle $S$ can be finished also in other than starting inter-depot, (either central warehouse can be assumed to serve as inter-depot), but the number of incoming and outcoming vehicles of type $S$ must be identical.

\section{Parameters}

D - matrix of minimal distances between all the pairs of nodes

$\mathbf{b}-$ vector of demands

$g_{L}$ - capacity of the vehicle $L$

$g_{S}-$ capacity of the vehicle $S$

$c_{L}-$ cost per unit of the vehicle $L$

$c_{S}-$ cost per unit of the vehicle $S$.

\section{Heterogeneous Fleet Vehicle Routing Problem with Selection of Inter-Depots - Model}

\section{Variables}

$x_{i j} \in\{0,1\}, i, j=1,2, \ldots n$ - binary variables, that represent the use of corresponding edge by vehicle $L$ : If the edge $(i, j)$ is on the route of the vehicle $L x_{i j}=1$, otherwise $x_{i j}=0$.

$y_{i j} \in\{0,1\}, i, j=1,2, . . n$ - binary variables, that represent the use of corresponding edge by vehicle $S$ : If the edge $(i, j)$ is on the route of the vehicle $S y_{i j}=1$, otherwise $y_{i j}=0$.

$y y_{i j} \geq 0, \quad i, j=1,2, . . n$ - non-negative variables, that represent the cumulative amount of unloaded goods transported on the edge $(i, j)$. 


\section{Č. Zuzana $E T \quad A L$.}

$u_{i} \geq 0, i=1,2, \ldots n$ - non-negative variables, that represent the cumulative demand of the individual nodes on the route of vehicle $L$ (including eventual distribution with a vehicles $S$ from corresponding node).

$z_{i} \geq 0, i=1,2, \ldots n$ - non-negative variables, that represent the cumulative demand of the individual nodes on the route of vehicle $S$.

$w_{i} \geq 0, i=2,3, \ldots n$ - non-negative variables, that represent the quantity of goods that is distributed from corresponding node by vehicle $S$.

$q_{i} \geq 0, i=1,2, \ldots n$ - non-negative variables, that represent the quantity of imported goods to the corresponding node by a vehicle $L$.

\section{Starting values of variables}

$$
\begin{aligned}
& x_{11}=1 \\
& x_{i i}=0, \quad i=2,3, \ldots n \\
& u_{1}=0 \\
& z_{1}=0
\end{aligned}
$$

The model can be interpreted as follows:

$$
f(\mathbf{X}, \mathbf{Y}, \mathbf{Y Y}, \mathbf{u}, \mathbf{z}, \mathbf{w}, \mathbf{q})=c_{L} \sum_{i=1}^{n} \sum_{j=1}^{n} d_{i j} x_{i j}+c_{S} \sum_{i=1}^{n} \sum_{j=1}^{n} d_{i j} y_{i j} \rightarrow \min
$$

Objective function ensures minimization of total transportation costs for vehicles $L$ and vehicles $S$.

$$
\sum_{i=1}^{n} x_{i j} \leq 1, \quad j=2,3, \ldots n, i \neq j
$$

The equations represent that the vehicle $L$ comes to each node (except the centre) no more than once.

$$
\sum_{j=1}^{n} x_{i j} \leq 1, \quad i=2,3, \ldots n, i \neq j
$$

The equations represent that the vehicle $S$ leaves to each node (except the centre) no more than once.

$$
\sum_{j=1}^{n} x_{i j}=\sum_{j=1}^{n} x_{j i}, \quad i=1,2, \ldots n, i \neq j
$$

The equations represents that the number of arrivals and exits of vehicle $L$ into and from the node is identical.

$\left(u_{i}+q_{j}-u_{j}\right) \cdot x_{i j}=0, i=1,2, \ldots n, j=2,3, \ldots n, i \neq j$

The equations provide the accumulation of the load of the vehicle $L$.

$u_{i} \leq g_{L}, i=2,3, \ldots n$

The equations represent that the capacity of vehicle $L$ must not be exceeded.

$$
\sum_{i=1}^{n} q_{i}=\sum_{i=1}^{n} b_{i}
$$

The equation represents that the demand of all nodes have to be met.

$$
\left(1-\sum_{j=1}^{n} x_{i j}\right) . b_{i} \leq z_{i}, i=2,3, \ldots n
$$

The equations provide the balance of variables (starting values for a vehicle $S$ ) in the case node serves as an inter-depot.

$$
-\left(\sum_{\substack{i=1 \\ i \neq j}}^{n} x_{i j}\right)+\left(\sum_{i=1}^{n} y_{j i}\right) \geq 0, j=2,3, \ldots n
$$

The equations represent the self-service of the node, in the case the node is on the route of a vehicle $L$.

$\sum_{j=1}^{n} y_{i j} \geq 1, \quad i=1,2, \ldots n$

The equations represent that each node must be served.

$\sum_{j=1}^{n} y_{i j}=\sum_{j=1}^{n} y_{j i}, \quad i=1,2, \ldots n, i \neq j$

The equations represent that the number of incoming and the number of outcoming routes in and out of the node is identical.

$$
\begin{aligned}
& \left(z_{j}+b_{i}-z_{i}\right) \cdot y_{i j} \cdot\left(1-\sum_{s=1}^{n} x_{s j}\right)=0, i=1,2, \ldots n, \\
& j=2,3, \ldots n, i \neq j
\end{aligned}
$$

The equations provide the accumulation of the load of the vehicle $S$.

$z_{i} \leq g_{S}, i=1,2, \ldots n$

The equations represent that the capacity of vehicle $S$ must not be exceeded

$$
w_{i}=q_{i}-b_{i} \cdot \sum_{j=1}^{n} x_{i j}, \quad i=2,3, \ldots n
$$

The equations represent the balance of quantity of goods of vehicle $S$.

$$
\sum_{i=1}^{n} y y_{j i} \cdot \sum_{\substack{i=1 \\ i \neq j}}^{n} x_{i j}=w_{j}, \quad j=2,3, \ldots n
$$

The equations provide that the amount of goods for vehicles $S$ cannot exceed the quantity of goods that is transported into the node by a vehicle $L$.

$$
y y_{j i} \cdot y_{j i}=z_{i}, i, j=1,2, \ldots n, i \neq j
$$

The equations provide the balance of cumulative number of unloaded goods transported on the edge $(i, j)$.

$$
\begin{aligned}
& y_{i j} \in\{0,1\}, i, j=1,2, \ldots n \\
& x_{i j} \in\{0,1\}, \quad i, j=1,2, \ldots n \\
& y y_{i j} \geq 0, \quad i, j=1,2, \ldots n \\
& u_{i} \geq 0, \quad i=1,2, \ldots n \\
& w_{i} \geq 0, \quad i=2,3, \ldots n \\
& z_{i} \geq 0, \quad i=1,2, \ldots n \\
& q_{i} \geq 0, \quad i=1,2, \ldots n
\end{aligned}
$$

\section{Empirical Results}

The principle of heterogeneous fleet vehicle routing problem with selection of inter-depots is illustrated on 


\section{Č. Zuzana $E T \quad A L$.}

three demonstrative instances. Firstly, cost per vehicle $L$ and vehicle $S$ were considered in proportion ten to six, $L(10), S(6)$. Later, in order to demonstrate the model functionality, it was considered to set significantly higher costs per vehicle $L$, (value 100) and to set significantly lower costs per vehicle $S$ (value 1). This should lead to preference of vehicles $S$ (if it is possible to fulfill the demands due to the restriction limit of the vehicle). In the third case, high cost for vehicle $S(100)$ and low cost for vehicle $L$ (1) were preferred, which should essentially results in the preference of vehicle $L$, i.e. the model works as classical CVRP.

Model input data, used in all three demonstrative instances:

$n-$ number of nodes $=10$

$\mathbf{b}-$ vector of demands $=(0,5,2,2,20,10,6,3,9,10)$

$g_{L}$ - capacity of the vehicle $L=50$

$g_{S}$ - capacity of the vehicle $S=15$

D - matrix of minimal distances between all the pairs of nodes (Table 1)

Table 1

\begin{tabular}{|r|r|r|r|r|r|r|r|r|r|r|}
\hline $\mathbf{D}$ & 1 & 2 & 3 & 4 & 5 & 6 & 7 & 8 & 9 & 10 \\
\hline 1 & 0 & 111 & 48.5 & 216.7 & 82.5 & 207.8 & 40.8 & 182.5 & 105.1 & 118.8 \\
\hline 2 & 111 & 0 & 77.5 & 301.6 & 33.5 & 125.9 & 151.8 & 80 & 78.9 & 121.9 \\
\hline 3 & 48.5 & 77.5 & 0 & 265.2 & 67 & 169.2 & 89.3 & 137.5 & 132.2 & 145.9 \\
\hline 4 & 216.7 & 301.6 & 265.2 & 0 & 273.1 & 424.5 & 182.1 & 374.5 & 259.1 & 272.8 \\
\hline 5 & 82.5 & 33.5 & 67 & 273.1 & 0 & 151.4 & 123.3 & 105 & 79.1 & 93.4 \\
\hline 6 & 207.8 & 125.9 & 169.2 & 424.5 & 151.4 & 0 & 248.6 & 91.5 & 184.4 & 228.1 \\
\hline 7 & 40.8 & 151.8 & 89.3 & 182.1 & 123.3 & 248.6 & 0 & 223.3 & 145.9 & 159.6 \\
\hline 8 & 182.5 & 80 & 137.5 & 374.5 & 105 & 91.5 & 223.3 & 0 & 117.9 & 161.6 \\
\hline 9 & 105.1 & 78.9 & 132.2 & 259.1 & 79.1 & 184.4 & 145.9 & 117.9 & 0 & 46.2 \\
\hline 10 & 118.8 & 121.9 & 145.9 & 272.8 & 93.4 & 228.1 & 159.6 & 161.6 & 46.2 & 0 \\
\hline
\end{tabular}

For each demonstrative instance the input data were modified in different cost adjustments:

1) Proportional costs adjustment:

$c_{L}-$ cost per unit of the vehicle $L=10$

$c_{S}-$ cost per unit of the vehicle $S=6$

2) The higher cost of operating a large capacity vehicle compared to a small capacity vehicles:

$c_{L}$ - cost per unit of the vehicle $L=100$

$c_{S}-$ cost per unit of the vehicle $S=1$

3) The higher cost of operating a small capacity vehicles compared to a large capacity vehicle:

$c_{L} \quad$ - cost per unit of the vehicle $L=1$

$c_{S}-$ cost per unit of the vehicle $S=100$

Proposed problems were solved using software GAMS.

Solutions of the model for various demonstrative instances:

1. Instance with proportional cost values $\left(c_{L}=10, c_{S}=\right.$

6). Computed value $f=9103.8$. Solution is depicted on Figure 1, dashed lines represent the transportation by vehicle $L$, and full line represents the route of vehicles $S$.

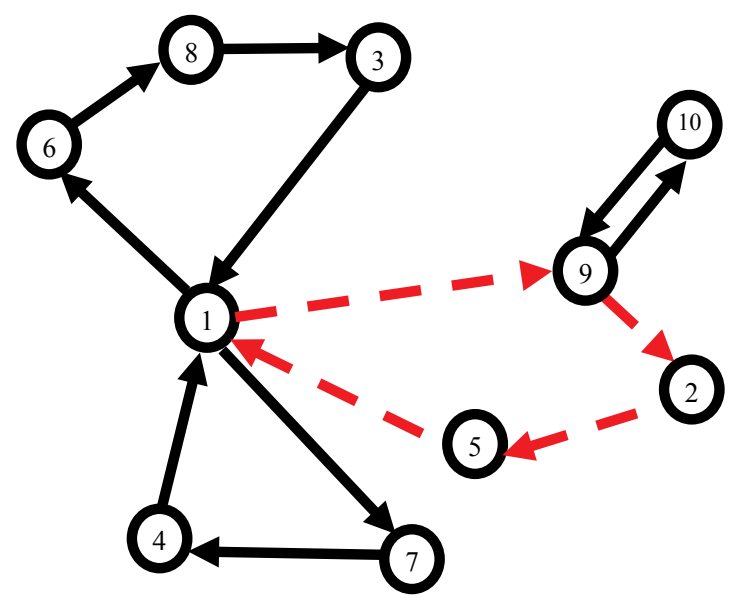

Figure 1

2. Instance with significantly higher cost of operating vehicle $L\left(c_{L}=100, c_{S}=1\right)$. Computed value $f=$ 17757.5. Solution is depicted on Figure 2, dashed lines represent the route of vehicle $L$ and full lines represent the route of vehicles $S$. The value of objective function $f$ has significantly increased. It is caused by the fact that delivery cannot be made only by vehicle type $S$ because of the demand of node 5 exceeds the capacity of the vehicle $S$. At the same time, assumption that the vehicle $S$ can finish its route in other as in starting inter-depot is met, however the number of incoming and outgoing vehicles of type $S$ must be identical (node 1 and 5).

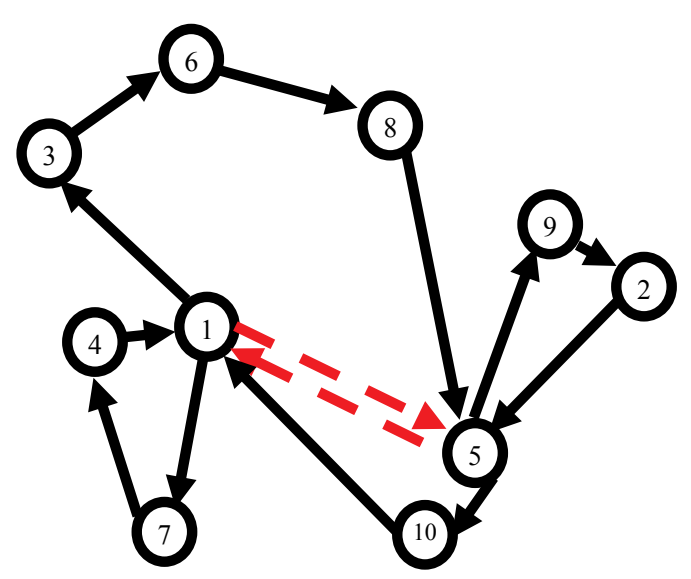

Figure 2

3. Case with significantly higher cost of operating vehicles $S\left(c_{L}=1, c_{S}=100\right)$. Computed value $f=$ 1152.2. Solution is depicted on Figure 3. Dashed lines represent the transportation by $L$ type vehicle, 
the vehicles $\mathrm{S}$ are not in use (as in classical CVRP).

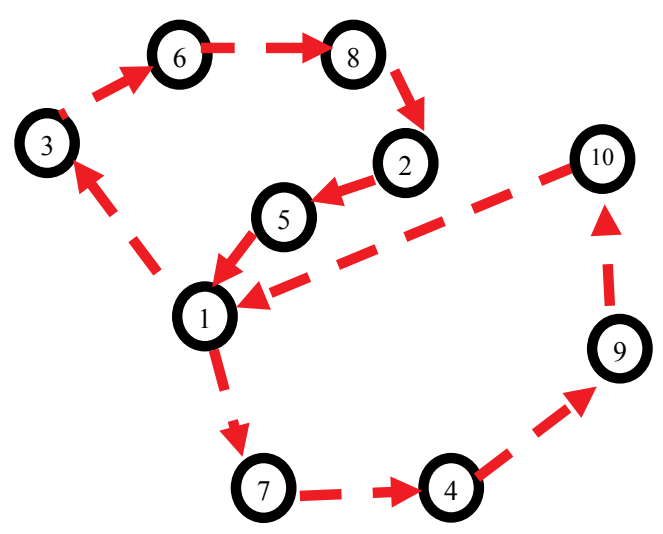

Figure 3

\section{Conclusion}

The paper is focused on the heterogeneous fleet vehicle routing problem with selection of inter-depots. It involves not only optimization of distribution on two stages, but also the choice between two types of vehicles based on cost optimization. The model deals with one central warehouse and $n-1$ delivery points (each of them can serve as an inter-depot). In the central warehouse, large capacity vehicles $L$ are available. Small capacity vehicles $S$ are available at the endpoints and also at the central warehouse.

The solution was presented on three demonstrative instances. The first case is close to real ratio of vehicle costs (proportional cost per vehicle $L$ (10) and vehicle $S$ (6)). Furthermore, also specific cases with extreme cost ratios have been considered, which should verify a "reasonable" behavior of the model. Obtained results confirm the feasibility of usage of the model which allows utilization of the existing storage capacities at delivery points (inter-depots), and could lead to a more efficient distribution.

\section{Acknowledgements}

This paper is supported by the Grant Agency of Slovak Republic - VEGA, grant no. 1/0104/12 „Modeling supply chain pricing policy in a competitive environment".

\section{REFERENCES}

[1] N. R. Achuthan, L. Caccetta and S. P. Hill, "An Improved Branch-and-Cut Algorithm for the Capacited Vehicle Routing Problem", Transportation Science, Vol. No. 37, 2003, pp. 153-169.
[2] Z. Čičková, I. Brezina and J. Pekár, "A memetic algorithm for solving the vehicle routing problem", In Mathematical methods in Economics 2011, 29th international conference, Praha, Professional Publishing, 2011, pp. 125-128.

[3] I. Brezina, Z. Čičková and J. Pekár, "Evolutionary approach as an alternative method for solving the vehicle routing problem", Economic review, Vol. 41, No. 2, 2012, pp. 137-147.

[4] Z. Čičková, I. Brezina and J. Pekár, “Alternative method for solving traveling salesman problem by evolutionary algorithm", Management information systems. No. 1, 2008, pp. 17-22.

[5] I. Brezina, Z. Čičková and J. Pekár, "Application of evolutionary approach to solving vehicle routing problem with time windows", Economic review, Vol. 38, No. 4, 2009, pp. 529-539.

[6] J. Pekár, I. Brezina, Z. Čičková and M. Reiff, "Modelovanie rozmiestňovania recyklačných centier “, EKONÓM, Bratislava, 2012.

[7] Z. Čičková, I. Brezina and J. Pekár, "SOMA for solving the vehicle routing problem with time windows “, In Zbornik radova : SYM-OP-IS 2008, Soko Banja. Beograd, 2008, pp. 305-308.

[8] J. F. Bard, G. Kontoravdis and G. Yu, “A Branch-and-Cut Procedure for the Vehicle Routing Problem with Time Windows". Transportation Science, Vol. 36, 2002, pp. 250-269.

[9] D. Burchett and E. Campion, "Mix Fleet Vehicle Routing Problem - An Application of Tabu Search in the Grocery Delivery Industry". University of Canterbury, New Zealand, 2002.

[10] S. Irnich, "A Multi-Depot Pickup and Delivery Problem with a Single Hub and Heterogenous Vehicles". European Journal of Operational Research, Vol. 122, No. 1, 2000, pp. 310-328.

[11] B. L. Golden, A. A. Assad, L. Levy and F. G. Gheysens, "The feet size and mix vehicle routing problem", Computers \& Operations Research, Vol. 11, 1984, pp. 49-66.

[12] A. Subramanian, P. H. V. Penna, E. Uchoa and L. S. Ochi, "A hybrid algorithm for the Heterogeneous Fleet Vehicle Routing Problem", European Journal of Operational Research, Vol. 221, 2012, pp. 285-295

[13] J. Pekár, I. Brezina and Z. Čičková, “Two-stage vehicle routing problem with dynamic selection of entrepots", In EURO 2012 : 25th European conference on operational research, 8-11 July 2012, Vilnius, Lithuania, 2012, pp. 124-125. 